$41(2) \mid 2012$

Varia

\title{
François Chevalier (1914-2012)
}

\section{Véronique Hebrard}

\section{(2) OpenEdition}

Journals

Édition électronique

URL : http://journals.openedition.org/bifea/685

DOI : 10.4000/bifea.685

ISSN : 2076-5827

\section{Éditeur}

Institut Français d'Études Andines

\section{Édition imprimée}

Date de publication : 1 juillet 2012

Pagination : 324-331

ISSN : 0303-7495

\section{Référence électronique}

Véronique Hebrard, «François Chevalier (1914-2012)», Bulletin de l'Institut français d'études andines [En ligne], 41 (2) | 2012, mis en ligne le 01 décembre 2012, consulté le 07 décembre 2020. URL : http:// journals.openedition.org/bifea/685; DOI : https://doi.org/10.4000/bifea.685

\section{(c) $(1) \odot$}

Les contenus du Bulletin de l'Institut français d'études andines sont mis à disposition selon les termes de la licence Creative Commons Attribution - Pas d'Utilisation Commerciale - Pas de Modification 4.0 International. 


\section{FRANÇOIS CHEVALIER}

\section{4-2012}

Le 6 juin 2012 François Chevalier nous quittait pour un ultime voyage, lui qui les aimait tant. La dernière fois que je l'ai vu, c'était à son domicile parisien de I'avenue Ledru Rollin, au début du printemps, après m'être inquiétée de ne pas avoir reçu de ses nouvelles par téléphone depuis un moment déjà, ce qui ne lui ressemblait pas. L'œil toujours vif et content de pouvoir partager un moment avec moi, il me parut moins alerte que la dernière fois qu'il était venu à la Sorbonne, juste avant les vacances de Noël, où il aimait tant venir passer quelques heures au Cralmi pour se mettre au courant des nouveautés, emprunter un ouvrage et discuter de l'Amérique latine.

Depuis lors, j'avais su par Josèphe son épouse qu'il était très fatigué et qu'il avait de plus en plus de mal à faire sa promenade quotidienne, lui qui fut un marcheur (et un skieur) hors pair, et pour qui le voyage, sous toutes ses formes, fut comme une devise, que ce soit pour accomplir son métier d'historien, pour découvrir des lieux et des monuments (les églises romanes qu'il affectionnait tout particulièrement) ou pour aller à la rencontre des autres.

Car le déplacement est sans doute l'un des termes qui lui sied le mieux, avec I'observation. Qu'il s'agisse des multiples déplacements qu'il a effectués en France pour la réalisation de son mémoire de l'École des Chartes, puis de ceux faits à 
l'étranger. En Espagne tout d'abord, où, de 1941 à 1945, il est élève de l'École des Hautes Études Hispaniques (Casa de Velázquez) à Madrid, d'où il se rend à Séville pour travailler aux archives et donner quelques cours. Puis de l'Espagne, après un bref retour en France, au Mexique où il restera de 1946 à 1962 et qu'il sillonnera en tous sens, ainsi que la plupart des pays de la zone, notamment andine lorsqu'il devient directeur de l'IFEA entre 1962 et 1966 (alors qu'il est en poste à l'université de Bordeaux). En Espagne à nouveau, de 1967 à 1979. Enfin, en tant que titulaire de la première chaire d'histoire de l'Amérique latine à Paris intra muros, à partir de 1980, il n'a cessé, pratiquement jusqu'à sa mort de parcourir l'Amérique latine dès que l'occasion lui en a été offerte, pour poursuivre son œuvre d'observation et de réflexion.

Mais il y a également les déplacements, plus symboliques et non moins déterminants, qu'il a effectués pour la réalisation des ses travaux d'historien. II n'a eu de cesse en effet de déplacer son regard, en empruntant également les chemins de l'ethnologie et de la littérature, pour mieux rendre compte de ses objets d'études, mais aussi de l'exercer à travers la focale de son appareil photo puis de sa caméra.

Il a tiré de ses déplacements un rapport empathique avec les différents terrains de ses études, les arpentant avec le vif désir de s'imprégner des espaces et de ceux qui les occupaient, nourri en cela de sa formation de géographe à Grenoble aux côtés de Raoul Blanchard, et des leçons de son « maître » Marc Bloch. Cette proximité physique avec le terrain, forgée au cours des innombrables déplacements qu'il a effectués avec les moyens de locomotion les plus diversifiés (du cheval, à la moto, en passant par les petits avions qu'il emprunta lorsqu'il accepta à son arrivée au Mexique en 1946 de porter la correspondance diplomatique pour les pays d'Amérique centrale qui transitait par México), m'a fait penser en de multiples occasions aux écrits de Pierre Sansot sur la marche, la lenteur, qu'il a mis bien souvent en rapport avec le travail de l'écriture et celui de l'enseignant qui doit savoir cheminer et se laisser aller à un certain vagabondage pour permettre à la pensée d'advenir' . Pierre Sansot qui enseigna la philosophie et l'anthropologie à I'université Pierre Mendès-France de Grenoble...

Ce n'est pas le lieu ici de rendre compte de son parcours et de son œuvre, qui furent, I'un et l'autre denses, fructueux, à l'origine de nombreux renouveaux tant en ce qui concerne la gestion d'établissements tels que ceux qu'il a dirigés, ses enseignements, qu'au niveau strictement historiographique. Ce serait même une gageure tant ils furent riches, féconds et tant ils s'inscrivent dans l'espace euroaméricain, et dans le temps...

Rappelons que François Chevalier étudie donc tout d'abord la géographie à I'Université de Grenoble. Il en garde notamment une passion pour les « paysages » que I'on retrouve dans son mémoire de l'École des Chartes (mémoire d'histoire rurale déjà sur la région Vienne en France, sur d'anciennes villas romaines) ; École

1 Il l'évoque avec particulièrement d'acuité dans le 19ème chapitre de Chemins aux vents, intitulé «Marcher, parler, écrire, lire » (Sansot, 2002 : 283-291). 
des Chartes où il est élève de 1936 à 1939 (et où il fit la rencontre de Marc Bloch). Mais passion qui sera également au cœur de sa thèse sur la formation des grands domaines au Mexique au XVle et XVIle qu'il commence en 1941 (suite à une autre rencontre décisive, avec Robert Ricard au Maroc où il est allé lui rendre visite) alors qu'il est en Espagne en tant que pensionnaire de la Casa de Velázquez. Cette thèse, plusieurs fois rééditées, entreprise sous la direction de Marc Bloch fondateur de l'École des Annales avec Lucien Febvre, marque un tournant majeur dans I'historiographie mexicaniste et au-delà, dès lors qu'elle appréhende ces grandes propriétés terriennes comme une totalité et dans une démarche pluridisciplinaire et en examine toutes les facettes (économique, agraire, juridique, humaines, sociales, politique). Surtout, outre la consultation des archives tout d'abord à Séville, ce travail s'effectue sur le terrain dès 1947 lorsque François Chevalier part au Mexique comme boursier de I'IFAL, sous l'égide de Paul Rivet son fondateur, qui a pris le relais de Marc Bloc pour la direction de sa thèse, après son assassinat en 1944 par les Allemands à Lyon. Il entreprend alors de parcourir le pays (notamment avec sa fameuse Harley Davidson) pour y retrouver les traces matérielles de ces grandes propriétés qu'il photographie et observe ainsi que les villes et villages traversés et leurs habitants, dans le même temps qu'il rédige ses fameux carnets de voyages fourmillant d'informations et de réflexions notées de sa fine écriture, de dessins, de croquis.

Lors des très nombreux voyages qu'il réalise alors et dont témoignent ces carnets ainsi que ses photos et ses films, il y a également la volonté de connaître le Mexique, de comprendre à travers le présent certains éléments du passé, en particulier les relations entre Indiens et Gente de razón, rancheros, les conflits qui se sont noués à l'époque coloniale, notamment autour de la terre et de son exploitation différenciée ; quête du passé qu'il traque également, encore, à travers les paysages. Questionnements qui l'amèneront à réaliser un voyage demeuré à ses yeux emblématique : celui qu'il effectua, en grande partie à cheval, avec son compagnon de voyage et ami fidèle, I'historien Ernesto de la Torre à Ostula dans le bas Michoacán, fin 1947 d'abord, puis en mai 1948. Il n’y avait pas filmé, n'ayant pas de caméra à l'époque, mais il avait, par contre, pris, là encore, de très nombreuses photos. Cette expédition à Ostula l'avait en particulier marqué parce qu'il représentait une première et forte impression du contraste, du décalage existant entre le monde indien et celui beaucoup plus métissé des rancheros, et les relations conflictuelles que ces populations entretenaient alors. J'ai refait ce voyage, en 2001, avec une partie de l'équipe du Projet Chevalier, en allant sur ses traces, nous appuyant pour ce faire sur ses carnets de voyages et ses photos de l'époque pour retrouver les espaces et les lieux parcourus, les paysages et les gens².

C'est en 1949 qu'il soutient sa thèse à la Sorbonne devant un jury composé, outre de Paul Rivet, de Fernand Braudel, de Charles André Julien, de Robert Ricard et de Marcel Bataillon.

2 Documentaire réalisé en mars 2001 par Augustin Viatte, intitulé Destination Ostula : sur les traces de François Chevalier, Gédéon Productions, avec la participation de Mathias Gardet, Jorge Santiago et moi-même. 
II repart quelques mois plus tard à México, en juillet 1949, accompagné de Josèphe qu'il vient d'épouser, en tant que directeur de l'IFAL, poste qu'il occupera jusqu'en 1962. Malgré ses responsabilités, il continue de voyager, y compris dans les autres pays d'Amérique latine, qu'il photographie et filme sans relâche, du Pérou au Guatemala, en passant par la Bolivie et l'Équateur. De retour en France en 1962, il exerce de nombreuses fonctions universitaires. II sera successivement professeur d'histoire et de civilisation de l'Amérique latine à I'Université de Bordeaux de 1962 à 1966, et directeur scientifique de l'IFEA (Institut Français d'Études Andines) pendant ses vacances, ce qui lui permet de conserver un contact avec le terrain ; directeur de la Casa de Velázquez de 1967 à 1979, tout en étant nommé, en 1970 professeur à I'Université de Paris I, occupant ainsi la première Chaire d'histoire de I'Amérique latine à Paris. Il sera en charge de ce poste jusqu'en 1983 avant d'en devenir professeur émérite.

Notons que ce parcours personnel se forge dans un moment crucial pour I'historiographie, celui de la fondation de l'École des Annales dont l'Amérique devait être un laboratoire (c'est le sens du double appel de Lucien Febvre en 1929 et en 1949 dans Les Annales) dans cette volonté revendiquée de mobiliser l'ensemble des sciences sociales pour appréhender les sociétés. Et que François Chevalier s'efforcera d'incarner, tant dans sa pratique d'historien, que d'enseignant et de directeur d'institutions françaises dans les mondes ibériques. Qu'il s'agisse de son usage de la littérature notamment pour appréhender les mondes ruraux (mexicain et andin), de l'attention portée au droit pour la compréhension des mécanismes de la colonisation, des rencontres qu'il n'aura de cesse d'organiser à l'IFAL notamment lors des Mesas Redondas où se rendront Lucien Febvre, Paul Rivet, Marcel Bataillon, Fernand Braudel et tant d'autres, pour dialoguer avec leurs confrères mexicains et américains, mais aussi en Espagne où son groupe de recherches interdisciplinaires basé à Séville s'est voulu une mise en pratique de cette démarche des Annales fondée sur le croisement des disciplines et des espaces, mais aussi sur le comparatisme, qu'il ne cessa de mettre en œuvre, que ce soit dans la synchronie ou dans la diachronie.

Ce parcours est aussi infléchi par la guerre, celle d'Espagne tout d'abord, dont il verra les conséquences in situ et plus tard au Mexique, puis la Seconde guerre mondiale dont on sait de quelle façon elle va le meurtrir et avoir des répercussions déterminantes pour la suite de sa carrière, mais aussi pour tous ceux de sa génération, sur les deux rives de l'Atlantique.

Passeur, diplomate, chercheur, quelle que soit la casquette de François Chevalier, on sent toujours cette empathie pour les espaces et les hommes qu'il a côtoyés, ce goût du débat, du dialogue, dont il eu le souci de garder des traces matérielles et orales et qui constituent le magnifique fonds d'archives, de bibliothèques, de films et de photos qu'il a constitués au fil des années. Bibliothèques qui reflètent sa passion pour les livres, de la «Taverna de Curro », cette cave où à Séville en 1941, il se livre à l'inventaire et à la lecture passionnée des livres de Diego Angulo, à celle qu'il a constitué au fil des ans, riche de plus de 4000 titres, en passant par la bibliothèque Paul Rivet dont il fut le maître d'œuvre à l'IFAL. 


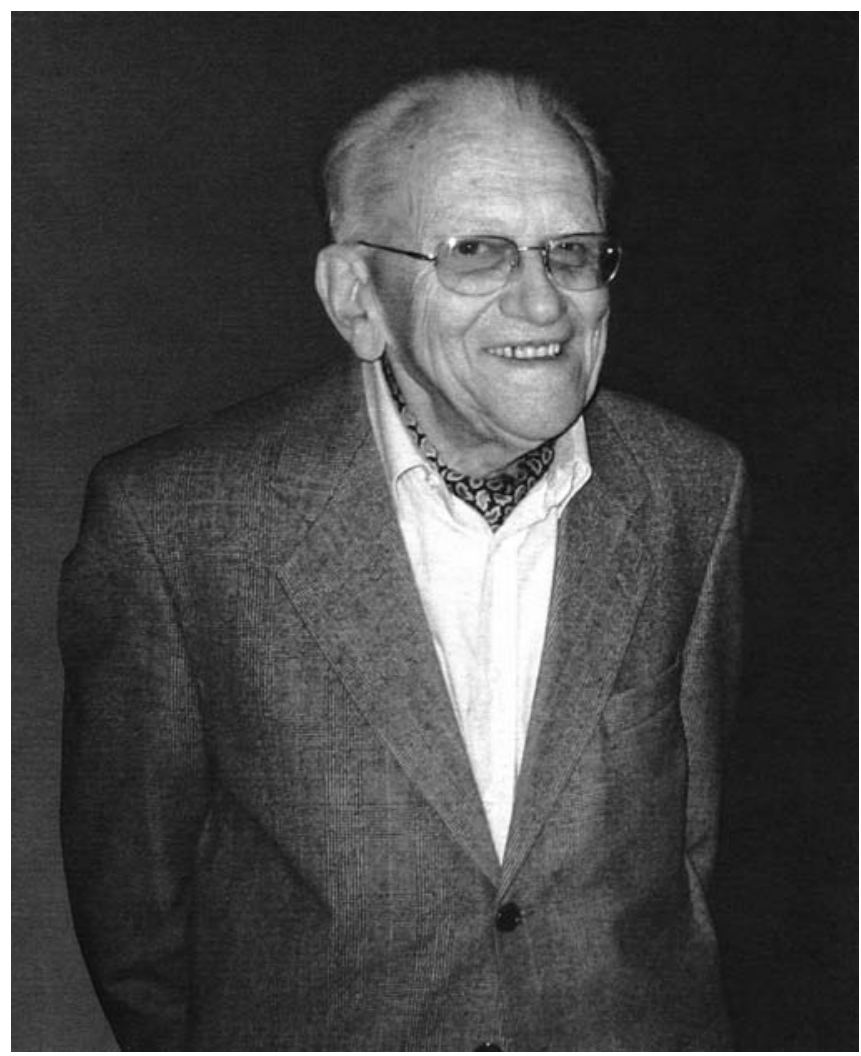

Films et photos nous renvoient à cette démarche pionnière qui constitue l'une de ses particularités : dès ses premiers séjours en Espagne il n'a eu de cesse de photographier ce qu'il observait (paysages, habitats, monuments, fêtes, scènes de la vie quotidienne...), puis de filmer, nous laissant en héritage un patrimoine visuel d'une extraordinaire richesses, qu'il nous reste encore très largement à explorer.

Nombreuses sont les images qui défilent devant mes yeux depuis la disparition de François Chevalier.

Images de travail tout d'abord, lorsque nous avons Mona Huerta, Mathias Gardet, Gérard Borras, Jorge Santiago et moi-même commencé le projet de recherche sur ses archives et sa bibliothèque, lorsque nous nous rendions chez lui pour faire I'inventaire des livres et des archives, pour réaliser des entretiens avec lui (qui ont été principalement réalisés par Jorge Santiago pendant près de deux ans) qui nous ont tant appris sur l'infra-ordinaire de sa pratique d'historien. Images encore de Monsieur Chevalier au CNRS lorsque nous visionnions ses films en super 8 qu'il n'avait pas vu depuis des années et que nous tentions de légender. Ces entretiens on permis, entre autres, de montrer que son cadre intellectuel est posé très tôt ; de même que ses filiations intellectuelles qui seront marquantes de par ceux qui les incarnent et en raison de leur permanence dans la structuration de sa pensée 
d'historien. Nous touchons, en effet dans le cadre de l'entretien, au sensible, à l'émotion, aux liens de confiance qui nécessairement se sont établis au fil des années et qui ont rendu l'échange possible, mais aussi la confidence.

Images également de I'appartement de François et Josèphe Chevalier où de chaque recoin, tiroir, malle, un trésor était susceptible de jaillir qu'il s'agisse de photos, de dossiers de travail, carnets de voyages de Monsieur Chevalier ou de ceux constitués par Mme Chevalier, bien plus archiviste parfois que son mari dans le soin qu'elle a mis a organiser certains documents, tandis que Monsieur Chevalier nous disait qu'il n'avait pas d'archives. Car ce sont tous ces matériaux qui, articulés à sa production scientifique, à ses enseignements, permettent de saisir à quel point sa démarche leur est consubstantielle. Ces matériaux qu'il a lui-même forgé, furent sans cesse mobilisés par François Chevalier pour construire et ordonner sa réflexion, les mettant sans cesse en regard les uns par rapport aux autres, conférant ainsi à chacun d'entre eux une valeur heuristique singulière.

Images de Monsieur Chevalier lorsque nous avons préparé le documentaire sur Ostula en 2001 : cette plongée dans un moment fondateur pour lui et qu'il nous demandait de refaire à sa place plusieurs décennies après, regardant ses photos, examinant avec nous les cartes pour retracer les itinéraires, avec ses carnets de voyage à l'appui ; son regard aiguisé des premières prises de vue et sur les « analyses » des uns et des autres; ses critiques aussi, toujours à-propos et constructives.

Images lors de la table ronde que nous avons organisée, en 2002, à la Maison de I'Amérique latine pour rendre compte des résultats du projet; images multiples de ces personnes mythiques qui se retrouvèrent, à la pause-déjeuner, autour d'une table dans un restaurant de Saint-Germain-des-Prés à refaire le monde et revivre des expériences du passé : Marianne Mahn Lot, Etienne Bloch (le fils de Marc Bloch), Claude Bataillon, Jean-Pierre Berthe...

Images lorsque j'ai travaillé avec lui à la réédition de sa thèse par les éditions Kathala en 2006 et où il a souhaité réactualiser une nouvelle fois non seulement la bibliographie, mais également l'introduction qu'il avait rédigée en guise de réponse aux débats que son travail avait suscité lors de sa première édition.

Images enfin de Monsieur Chevalier venant au Cralmi, jusqu'en décembre dernier donc, pour s'informer de la vie scientifique autour de l'Amérique latine, des dernières acquisitions du centre, de l'avancement de l'inventaire de ses archives et de ses livres.

Images plus personnelles de longues discussions chez les Chevallier, à Paris et dans I'Allier où nous avons plusieurs fois été accueillis avec cette chaleur et cette convivialité qui fit leur renommée au Mexique et ailleurs grâce à la sociabilité légendaire de Mme Chevalier et ses milliers de convives qui fréquentèrent leur « table», celle de leur domicile, mais aussi les fameuses mesas redondas de I'IFAL.

Discussions qui articulaient sans cesse le passé et le présent dans cette fidélité à I'histoire régressive de Marc Bloch ; qu'il s'agisse du monde rural, des pratiques sociales et politiques; discussions où la comparaison affleurait toujours dans cette 
curiosité qui était la sienne partout où il voyageait de « retrouver » des points de comparaison et que nous avions déjà remarquée dans ses carnets de voyages sur le Mexique (avec l'Espagne bien sûr, mais aussi avec I'Italie, le Maghreb...). Discussions également plus théoriques et méthodologiques sur I'histoire et les sources (pas seulement les archives), sur la nécessité de connaître son terrain au présent, de le parcourir. Histoire des gens et des structures, où l'apport des Annales et de Marc Bloch planait nécessairement.

Les unes et les autres ont nourri mon parcours d'historienne et ma connaissance des mondes américains mais aussi de la construction de l'américanisme de la première moitié du XXème siècle, et c'est une dette très grande que j'ai à son égard.

Indissociable de cette admiration intellectuelle pour l'historien, pour le directeur d'institutions prestigieuses auxquelles il a donné un souffle déterminant, je voudrais dire également mon affection pour la personne si discrète et si généreuse, toujours attentive, enfermée parfois dans ses blessures intimes et à propos desquelles il me livrait depuis peu des éléments, de façon orale mais aussi plus matérielle.

De nombreux hommages lui ont été rendus déjà au Mexique, en France, en Espagne, auxquels il avait le plus souvent participé. Et en ouverture desquels Bernard Lavallé pour celui de Bordeaux (Lavallé, 1990) et Ricardo Avila Palafox pour I'hommage mexicain (Ávila Palafox, 1992), ont mis en lumière de façon suggestive I'apport de François Chevalier non seulement pour I'historiographie mexicaine, mais également latino-américaine, et au-delà. Des publications et des colloques on été organisés autour du projet de recherches sur son patrimoine3, d'autres viendront sûrement.

Je terminerais par l'un des premiers qui lui fut en quelque sorte rendu à travers le compte-rendu que Lucien Febvre fit de sa thèse dans les Annales, à l'occasion de sa publication en 1952. Après avoir présenté l'ouvrage, les avancées qu'il comportait, il se réjouissait de la comparaison qu'il pourrait enfin permettre avec d'autres espaces et d'autres moments et terminait sur ces mots, ouverts sur I'avenir: «Félicitons le 'Conquérant' qui a quitté les douceurs de sa terre natale, pour s'en aller là-bas faire du bon travail et préparer les moissons futures » (Febvre, 1953 : 241).

A mon tour de lui rendre hommage et d'espérer que nous saurons porter loin et avec la même éthique et la même générosité les fruits, nombreux, de ces moissons.

3 Il y a la table ronde déjà mentionnée, qui a donné origine à Sur les traces d'un mexicaniste français. Constitution et analyse du fonds François Chevalier (Hébrard, 2005). Un colloque a également été organisé au Mexique en novembre 2007 : Coloquio sobre Las escuelas historiográficas de Francia y México, circulación, recepción y debates. Homenaje a François Chevalier (Cemca, IFAL, Université Paris I/Cralmi). 


\section{Références citées}

ÁVILA PALAFOX, R., 1992 - Introducción. In: Las Formas y las Políticas del Dominio Agrario. Homenaje a François Chevalier (R. Ávila Palafox, C. Martínez Assad \& J. Meyer, eds.) : 11-16 ; Guadalajara : Editorial Universidad de Guadalajara. Colección Fundamentos/Serie Laboratorio de Antropología.

FEBVRE, L., 1953 - Compte-rendu. Annales ESC, 8e année, n. ${ }^{\circ} 2$ : 273-241.

HÉBRARD, V. (ed.), 2005 - Sur les traces d'un mexicaniste français. Constitution et analyse du fonds François Chevalier, 328 pp. ; Paris : Karthala, collection Pollens.

LAVALLÉ, B., 1990 - Introduction. In : Structures et cultures des sociétés ibéro-américaines ; au-delà du modèle socio-économique. Colloque international en hommage au professeur Fr. Chevalier, 29-30 avril 1988 : 7-10 ; Paris : éd. du CNRS, collection de la Maison des pays ibériques.

SANSOT, P., 2002 - Chemins aux vents, 203 pp. ; Paris : Rivages poche.

Véronique HÉBRARD 